\title{
The PTEN/PI3K/Akt and Wnt// -catenin signaling pathways are involved in the inhibitory effect of resveratrol on human colon cancer cell proliferation
}

\author{
YING-ZI LIU ${ }^{1,2}, \mathrm{KE} \mathrm{WU}^{1,2}$, JUN HUANG ${ }^{1,2}$, YANG LIU $^{1,2}$, XIN WANG $^{1,4}$, ZI-JUN MENG $^{1,4}$, \\ SHUANG-XUE YUAN ${ }^{1,2}$, DONG-XU WANG ${ }^{1,2}$, JIN-YONG LUO ${ }^{3}$, GUO-WEI ZUO ${ }^{3}$, LIANG-JUN YIN ${ }^{4}$, \\ LIANG CHEN ${ }^{4}$, ZHONG-LIANG DENG ${ }^{4}$, JUN-QIN YANG ${ }^{1,2}$, WEN-JUAN SUN ${ }^{1,2}$ and BAI-CHENG HE ${ }^{1,2}$ \\ ${ }^{1}$ Chongqing Municipal Key Laboratory of Higher Education Institutions for Biochemistry and Molecular Pharmacology; \\ ${ }^{2}$ Department of Pharmacology, School of Pharmacy, Chongqing Medical University; ${ }^{3}$ Key Laboratory for Diagnostic \\ Medicine designated by the Chinese Ministry of Education, Chongqing Medical University; ${ }^{4}$ Department of \\ Orthopedic Surgery, Second Affiliated Hospital, Chongqing Medical University, Chongqing, P.R. China
}

Received January 16, 2014; Accepted March 11, 2014

DOI: $10.3892 /$ ijo.2014.2392

\begin{abstract}
Colon cancer is one of the most common malignancies and the treatments for colon cancer have been developed substantially in the last decades, but there is still a great clinical need to explore new treatment regimens due to the undesirable prognosis. In this investigation, we demonstrated the anti-proliferative and apoptosis-inducing activities of resveratrol (Res) in human colon cancer cells, and the possible mechanisms underlying these effects. We used crystal violet staining, flow cytometry and western blotting to validate the anti-proliferative and apoptosis-inducing effects of Res on HCT116 cells. A xenograft tumor model was used to confirm the anti-proliferative effects of Res. We employed polymerase chain reaction, western blotting, recombinant adenovirus and luciferase reporter assay to explore the possible mechanism(s) of action. We found that Res inhibits significantly the proliferation and promotes apoptosis in HCT116 cells, as well as inhibits the xenograft tumor growth of colon cancer. Res upregulates the expression of phosphatase and tensin homolog (PTEN) and decreases the phosphorylation of Akt1/2. The exogenous expression of PTEN inhibits the PI3K/Akt signal and promotes the anti-proliferative effects of Res in HCT116 cells, while knockdown of PTEN increases PI3K/Akt signal but reduces the anti-proliferative function of Res. The protein and mRNA expression of $\beta$-catenin are all decreased by Res concentration-dependently. Thus, our findings strongly suggest
\end{abstract}

Correspondence to: Professor Bai-Cheng He, Department of Pharmacology, Chongqing Medical University, 1 Yixueyuan Road, Yuzhong, Chongqing 400016, P.R. China

E-mail: hebaicheng99@yahoo.com

Key words: resveratrol, colon cancer, anti-proliferation, PTEN/ $\mathrm{PI} 3 \mathrm{~K} / \mathrm{Akt}, \mathrm{Wnt} / \beta$-catenin that the anti-proliferative effects of Res in human colon cancer cells may be mediated by regulating separately the PTEN/ $\mathrm{PI} 3 \mathrm{~K} / \mathrm{Akt}$ and $\mathrm{Wnt} / \beta$-catenin signaling.

\section{Introduction}

Colorectal cancer (CRC) is one of the most common malignancies. The incidence of colon cancer shows an increasing trend with tendency for younger age (1). The treatments of colon cancer including surgery resection or radiotherapy combined with chemotherapy (2). However, these treatments improved patient survival with the decreasing of life quality. Thus, there is an urgent clinic need to develop new treatment regimens for colon cancer.

Natural products are widely accepted as validated agents for many conditions (3-6). Many herbs or their components have already been used clinically as potential candidates for anticancer drugs, including anthocyanidins, catechins, quercetin and genistein $(7,8)$. A few studies and clinical epidemiologic studies indicated that red wine has apparent effect on reducing the risks of cardiovascular diseases and cancer $(9,10)$. Polyphenolic compounds are present at high levels in red wine, which may mainly contribute to the benefits of red wine.

Resveratrol (3,5,4'-trihydroxystilbene, Res), as a natural polyphenolic compound mostly from beans and grapes, was discovered in red wine by Siemann and Creasy in 1992 (11), and can be used as inhibitor for platelet aggregation, cardiacprotection and as anticancer agent $(10,12)$. It has been reported also that Res can inhibit diet induced obesity (13) inhibiting proliferation and promoting apoptosis in various tumor cells, such as colon cancer, breast cancer and prostate cancer cells in vitro and in vivo (14-20). For colon cancer cells, the anti-proliferation and apoptotic inducing effects of Res have already been validated, but the mechanism underlying these activities remains unclear.

PI3K/Akt signaling plays a critical role in modulating cell survival and apoptosis (21), it has been found over-activated in many cancers reducing apoptosis and promoting prolif- 
eration. The PI3K/Akt signaling was regulated by many other factors, one of the major negative regulators is phosphatase and tensin homologue (PTEN). PTEN was identified as a tumor suppressor $(22,23)$, which is often deleted or mutated in a variety of cancers at high frequency $(24,25)$. PTEN protein acts as a phosphatase specifically catalyzing the dephosphorylation of the 3-phosphate of the inositol ring in phosphatidylinositol $(3,4,5)$-trisphosphate (PIP3), leading to the biphosphate product PIP2. The dephosphorylation of PIP3 results in the inactivation of PI3K/Akt signal pathway because PIP3 is critical in activation of Akt (26).

$\mathrm{Wnt} / \beta$-catenin signaling pathway is important for cell proliferation and differentiation, its aberrant activation is another major cause of colon cancer. The Wnt/ $\beta$-catenin signaling can be upregulated by the phosphorylation of glycogen synthase 3 kinase $\beta$ (GSK3 $\beta)$, an important negative regulator for Wnt/ $\beta$-catenin signaling pathway, when PI3K/Akt signal is activated $(27,28)$.

Herein, we investigated the possible molecular mechanism underlying the proliferation inhibitory and apoptosis inducing activities of Res in colon cancer cells. We found that Res exhibits prominent anti-proliferation and apoptosis inducing activity in HCT116 cells. Mechanistically, we demonstrated that Res can upregulate the expression of PTEN and inhibit the activation of PI3K/Akt signaling pathway, as well as to inhibit the Wnt/ $\beta$-catenin signaling transduction, respectively. Our results support that Res can be used as anticancer agent alone or combined with other agents in colon cancer treatment.

\section{Materials and methods}

Reagents and cell culture. Resveratrol (Res) was purchased from Xi'an Haoxuan Biotechnology Co. Ltd., (Xi'an, China), and dissolved with dimethyl sulfoxide (DMSO). The aliquots were kept at $-20^{\circ} \mathrm{C}$. VO-OHpic was from Sigma-Aldrich (St. Louis, MO, USA). HCT116 cell line was kindly provided by Dr Bert Vogelstein (Johns Hopkins Oncology Center; Baltimore, MD, USA). For in vivo experiment, Res was prepared with $0.5 \%$ carboxymethylcellulose sodium (CMC$\mathrm{Na}$ ) as suspension. All antibodies were purchased from Santa Cruz Biotechnology, Inc. (Santa Cruz, CA, USA). Cells were maintained in the Dulbecco's modified Eagle's medium (DMEM) with $10 \%$ fetal bovine serum (FBS), $100 \mathrm{U} / \mathrm{ml}$ of penicillin, and $100 \mu \mathrm{g} / \mathrm{ml}$ of streptomycin at $37^{\circ} \mathrm{C}$ in $5 \% \mathrm{CO}_{2}$.

Crystal violet viability assay. Crystal violet assay was conducted as previously described (29). Experimentally, HCT116 cells were treated with indicated concentrations of Res. At 24,48 or $72 \mathrm{~h}$ after treatment, cells were carefully washed with cold phosphate-buffered saline (PBS, $4^{\circ} \mathrm{C}$ ) and stained with $0.5 \%$ crystal violet formalin solution at room temperature for 20-30 min. The stained cells were washed with water and air dried for imaging and quantification. For quantification, the crystal violet was dissolved in $20 \%$ acetic acid at room temperature for 20 min with shaking. The absorbance at $570 \mathrm{~nm}$ was measured (30).

Flow cytometry analysis for cell cycle and apoptosis. Cells were seeded into 6-well plates. For cell cycle analysis, cells were treated with different concentrations of Res or solvent for $48 \mathrm{~h}$.
Then, cells were washed with PBS, collected and washed with cold $\left(4^{\circ} \mathrm{C}\right) 70 \%$ ethanol followed by washing with $50 \%, 30 \%$ ethanol and PBS; incubated with $1 \mathrm{ml}$ of $20 \mathrm{mg} / \mathrm{ml}$ propidium iodide (PI) containing RNase $(1 \mathrm{mg} / \mathrm{ml})$ in PBS for $30 \mathrm{~min}$ followed by fluorescence activated cell sorting (FACS) assay. For apoptosis measure, cells were harvested after treated with different concentrations of Res for $48 \mathrm{~h}$. Then, cells were washed with PBS $\left(4^{\circ} \mathrm{C}\right)$, followed by incubating with Annexin V-EGFP and PI as the kit procedures (KeyGen Biotech Co. Ltd., Nanjing, China). Then, the cells were subjected to FACS assay.

Western blot assay. Cells were seeded in 6-well plates and treated with different concentrations of Res or solvent. At the scheduled time point, cells were lysed and the lysate were denatured by boiling for $10 \mathrm{~min}$. Total protein were separated by SDS-PAGE, transfered with polyvinylidene difluoride (PVDF) membrane, blocked in 10\% skimmed milk and probed with antibody against the target proteins. Finally, the images of target bands were developed with SuperSignal West Pico Chemiluminescent substrate.

RNA isolation and reverse transcription polymerase chain reaction $(R T-P C R)$. Cells were seeded in T25 flask and treated with different concentrations of Res. The total RNA was extracted with TRIzol reagents (Invitrogen, Carlsbad, CA, USA), followed by reverse transcriptional reaction to obtain the cDNA product. Finally, the cDNA products were used as PCR templates to detect the target gene expression. The primer sequences are available upon request.

Construction of recombinant adenoviruses for exogenous expression of PTEN, GFP and knockdown siRNA fragments for PTEN. Recombinant adenoviruses for exogenous expression of PTEN (Ad-PTEN) or GFP (Ad-GFP) were generated with the AdEasy system as previously described (31) as well as the recombinant adenoviruses expressing small interference RNA (siRNA) fragments for PTEN silence (Ad-siPTEN). Recombinant adenovirus expressing GFP was used as the vector control.

Xenograft tumor model of human colon cancer and histological evaluation. All animal experiments followed the guidelines of Institutional Animal Care and Use Committee of Chongqing Medical University (Chongqing, China). Athymic nude mice (female, 4-6 weeks old, 5/group) were from the animal center of Chongqing Medical University (Chongqing, China). HCT116 cells were cultured and resuspended in PBS for subcutaneous injection (1x10\%/injection) into the flanks of the athymic nude mice. The mice were treated with Res (50 or $150 \mathrm{mg} / \mathrm{kg}$ ) or the same volume of solvent through intragastric administration one week after cancer cell injection, once a day for four weeks. At the end of the 4th week, all nude mice were sacrificed, the tumor samples were retrieved and fixed in $10 \%$ formalin, and then embedded in paraffin. Serial sections of the embedded samples were stained with hematoxylin and eosin (H\&E).

Luciferase reporter assay. Firefly luciferase reporter assay was carried out as follows. Cells were seeded in T25 flasks and transfected with $3.0 \mu \mathrm{g}$ per flask of pTOP-Luc $(\beta$-catenin/Tcf4 

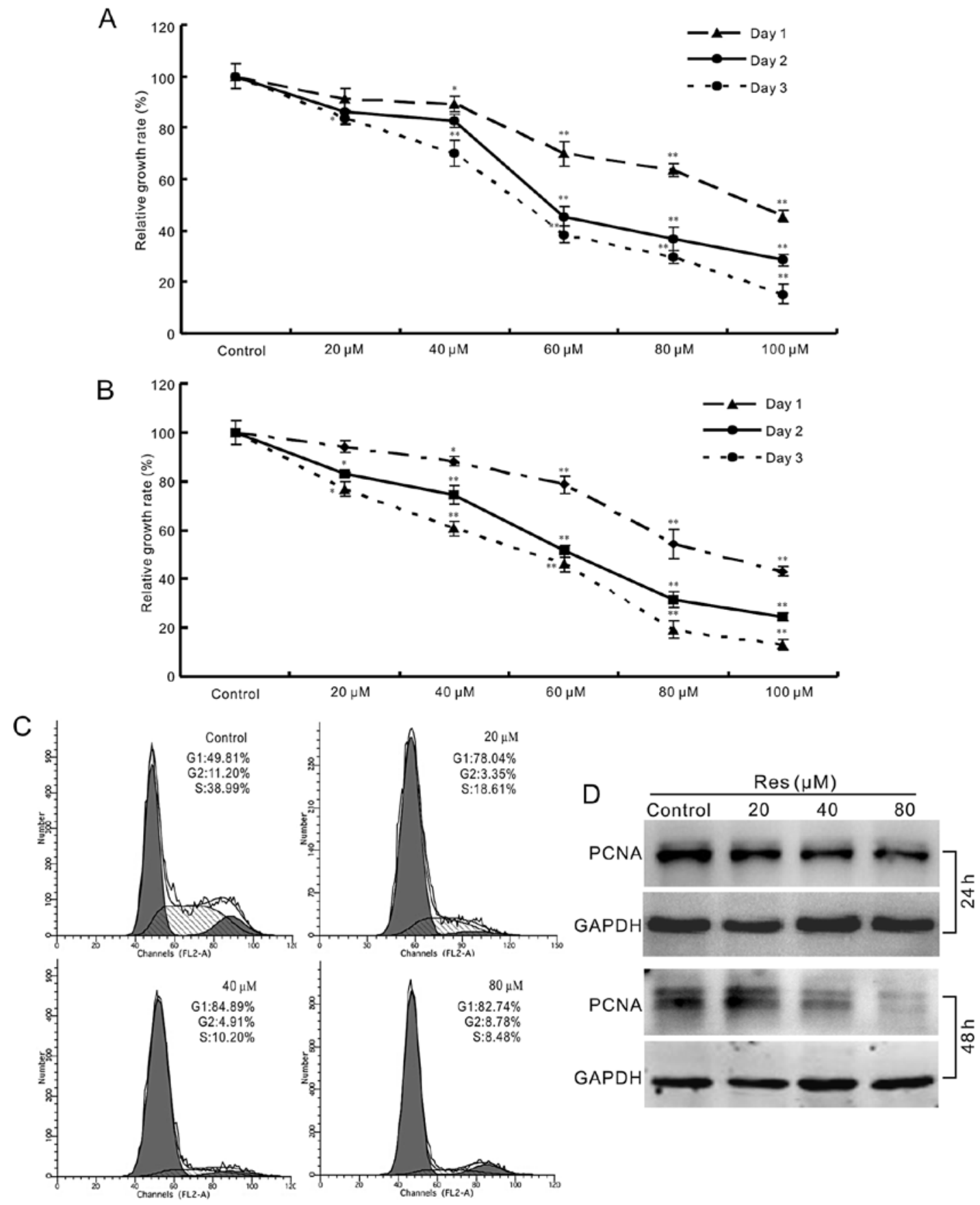

Figure 1. Resveratrol (Res) inhibits the proliferation of colon cancer cells. (A) Res inhibits the proliferation of HCT116 cells. Cells were seeded in 24-well plates and treated with different concentrations of Res or solvent, followed by crystal violet staining at the indicated time point. The crystal violet results were quantified as indicated in Materials and methods. $\mathrm{P}<0.05$ vs. control group; ${ }^{* *} \mathrm{P}<0.01$ vs. control group. (B) Res inhibits the proliferation of $\mathrm{SW} 480$ cells. This assay was carried out as described in (A). ${ }^{*} \mathrm{P}<0.05$ vs. control group; ${ }^{* *} \mathrm{P}<0.01$ vs. control group. (C) Res arrests the cell cycle at G1 phase in HCT116 cells. (D) Res decreases the protein level of PCNA in HCT116 cells. Each condition was done in triplicate.

responsive elements reporter plasmid) using Lipofectamine (Invitrogen). After $12 \mathrm{~h}$, cells were replated in 24-well plates and treated with different concentrations of Res or solvent as control. After $24 \mathrm{~h}$, cells were lysed and subjected to luciferase activity assays following the manual of the kit (Promega, Madison, WI, USA). Luciferase activity was normalized with total cellular protein concentrations of the samples. Each assay was done in triplicate.

Statistical analysis. All experiments were performed in triplicates and the results were repeated in at least two independent experiments. Statistical analysis of results was conducted using t-test (Microsoft Excel). Data are expressed as mean \pm standard deviation (SD). Statistical significance was set at $\mathrm{P}<0.05$.

\section{Results}

Resveratrol inhibits the proliferation in colon cancer cells. It has been reported that Res inhibits the proliferation of various cancer cells and modulates the processes of cancer (32). To investigate whether Res can affect the proliferation of human 
A
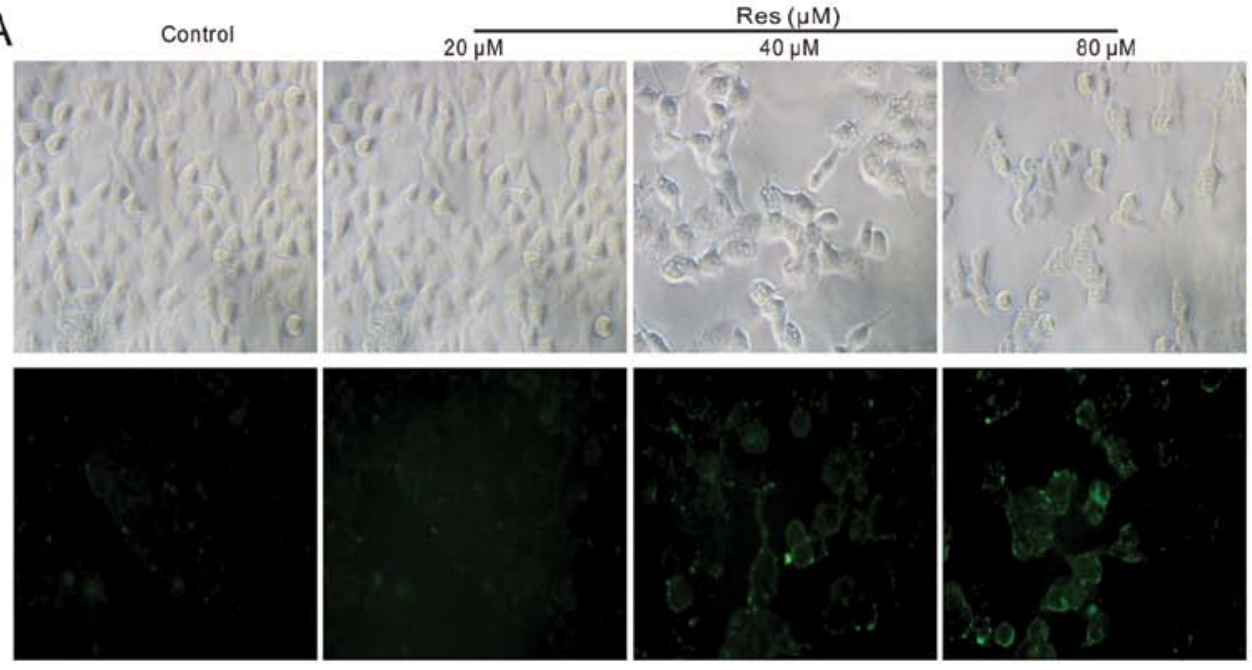

B
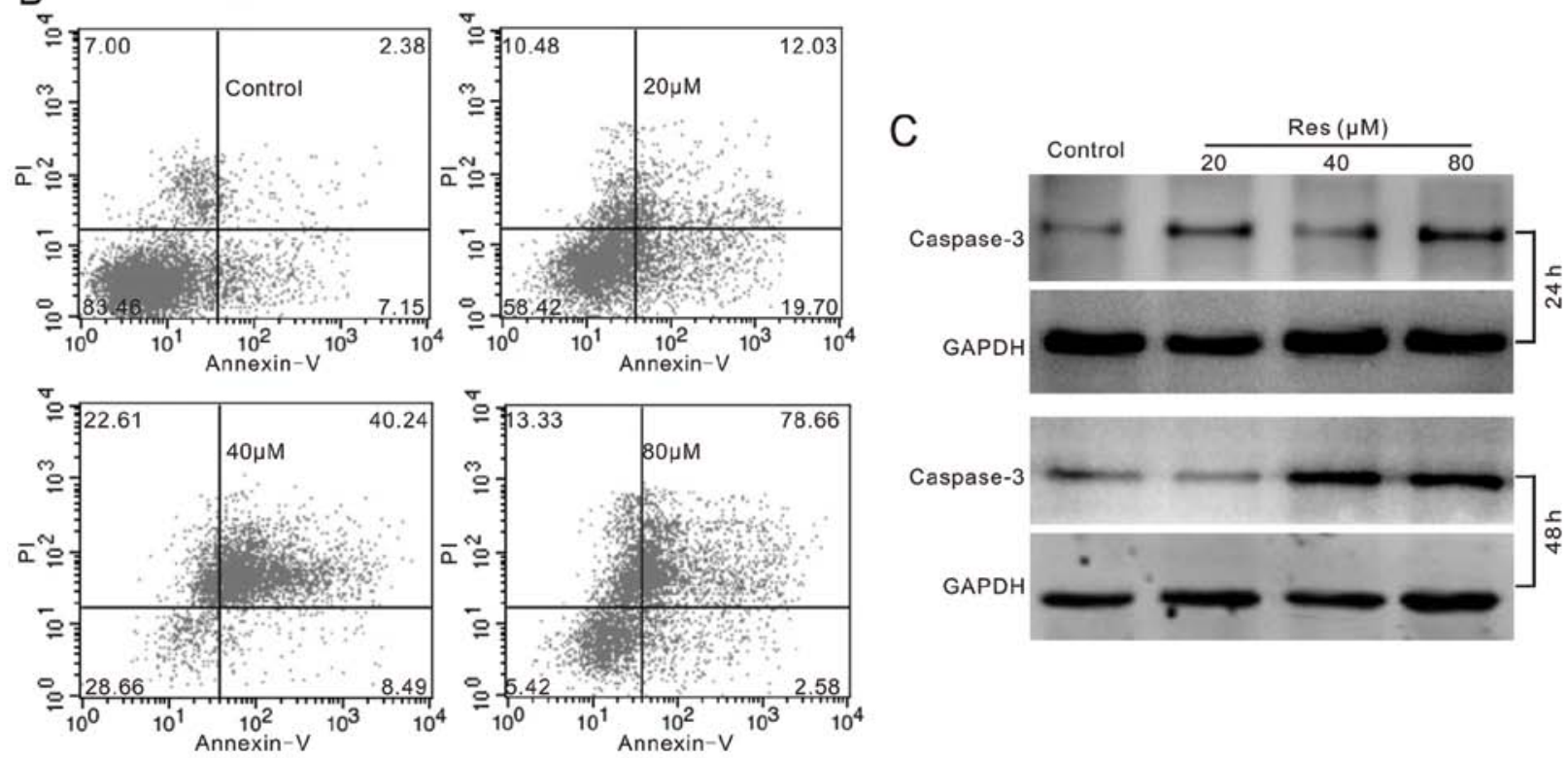

Figure 2. Resveratrol (Res) induces apoptosis in colon cancer cells. (A) Res induces apoptosis in HCT116 cells. Cells were seeded in 24-well plates and treated with different concentrations of Res for $48 \mathrm{~h}$, then the cells were washed with PBS and incubated with Annexin V-EGFP. Images were taken with a fluorescent microscope. (B) Res induces apoptosis in HCT116 cells. Cells were seeded in 6-well plates and treated with different concentrations of Res for $48 \mathrm{~h}$. Then, the cells were harvested and stained with Annexin V-EGFP and PI, followed by flow cytometry analysis. (C) Res increases the protein level of caspase-3 in HCT116 cells. Cells were seeded in 6-well plates and treated with different concentrations of Res. Total proteins were harvested after treating for 24 and $48 \mathrm{~h}$, followed by western blot assay as described in Materials and methods. GAPDH was used as loading control. Each condition was done in triplicate.

colon cancer, we analyzed the effect of Res on proliferation in human colon cancer cells. The results indicate that Res can inhibit the proliferation of HCT116 and SW480 cells concentration-dependently (Fig. 1A and B). Cell cycle analysis shows that Res can arrest the cell cycle at G1 phase in HCT116 cells (Fig. 1C). For further testing, we detected the effect of Res on the expression of proliferating cell nuclear antigen (PCNA) in HCT116 cells. The result shows that Res decreases the expression of PCNA concentration-dependently in HCT116 cells (Fig. 1D). These data demonstrate that Res is able to inhibit the proliferation of HCT116 cells.

Resveratrol induces apoptosis in colon cancer cells. Most anticancer agents have characteristics of apoptosis induction, so we tested whether Res could induce apoptosis in HCT116 cells. We employed Annexin V staining, FACS and western blotting to assay the effect of Res on apoptosis in HCT116 cells. The results show that Res can induce apoptosis clearly and concentration-dependently (Fig. 2A and B). Western blot assay results indicate that the protein level of caspase-3 increased substantially (Fig. 2C). These results strongly suggest that Res is a potent apoptosis inducer for human colon cancer cells.

Resveratrol inhibits tumor growth in a xenograft tumor model. The above evidence has proven that Res is a potent proliferation inhibitor agent for colon cancer cells. We next investigated the in vivo anticancer activity of Res with a xenograft tumor model for colon cancer. We injected $1 \times 10^{6}$ HCT116 cells into flanks of athymic nude mice. One week after injection, we treated the mice with intragastric administration of Res (50 or $150 \mathrm{mg} / \mathrm{kg}$ ), once a day for four weeks. 
A

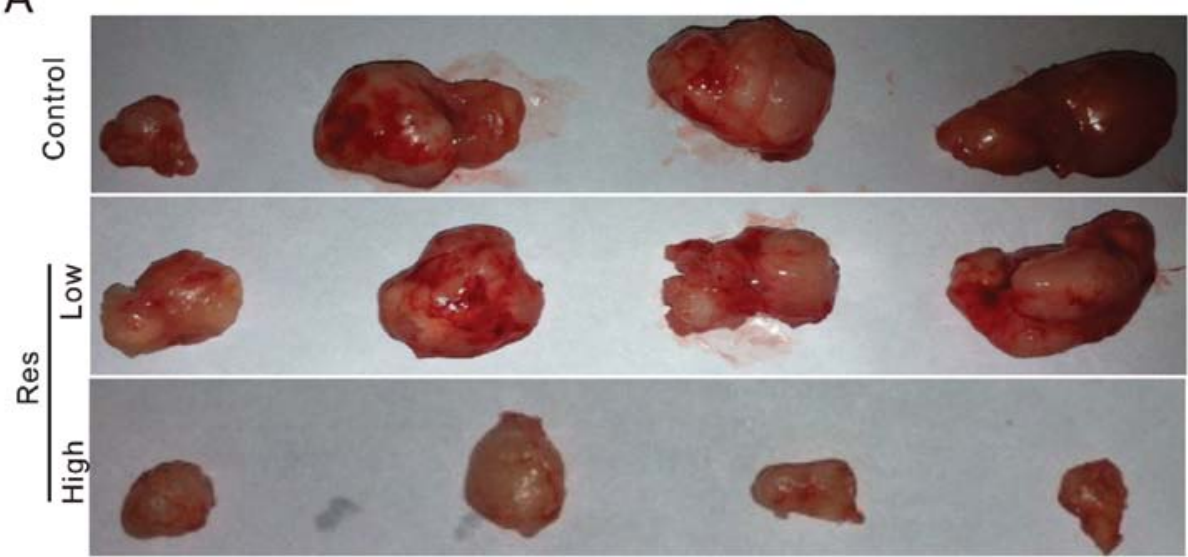

B
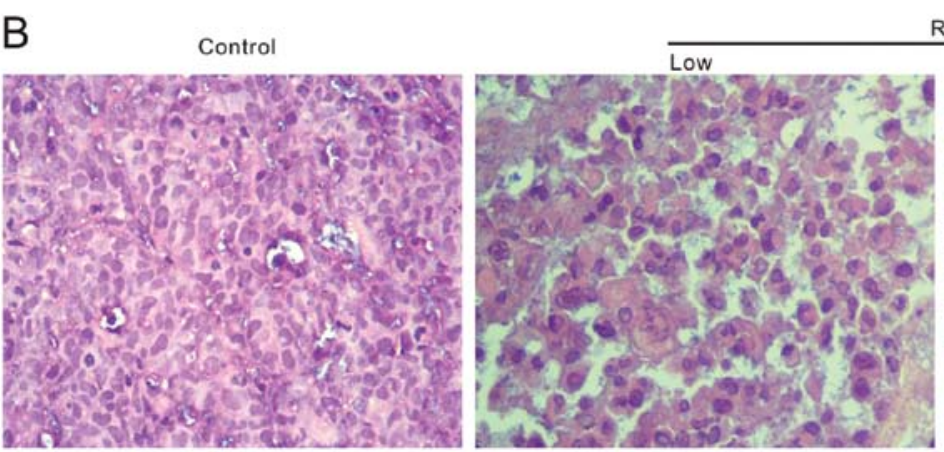

Res

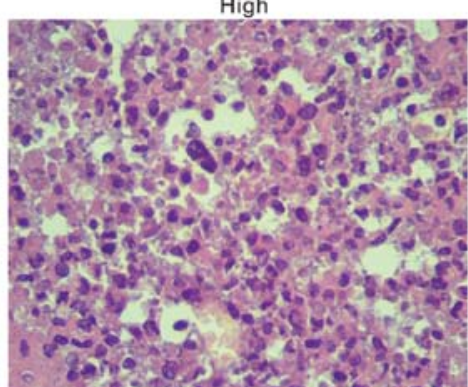

Figure 3. Resveratrol (Res) inhibits xenograft tumor growth of colon cancer. (A) Representative tumor masses from xenograft tumor assay. HCT116 cells were injected into the flanks of athymic nude mice (five mice per group, $1 \times 10^{6}$ cells per injection). Res was intragastrically administered one week after cancer cell injection ( 50 or $150 \mathrm{mg} / \mathrm{kg}$ ), once a day for four weeks. The mice were sacrificed at the end of the fourth week after injection, and the tumor masses were harvested for histologic analysis. (B) Histological examination of the xenograft tumor masses. Retrieved tumor masses were fixed, embedded and subjected to hematoxylin and eosin staining. Representative images are shown.

The results show that tumor masses from mice treated with Res are smaller than those of the control group (Fig. 3A). The H\&E staining results indicate that Res treated groups exhibited a decreased cellularity in tumor masses (Fig. 3B) suggesting that Res can inhibit tumor growth in vivo, although it can not eliminated tumors completely.

PTEN is involved in the anti-proliferation effect of resveratrol in colon cancer cells. PTEN, as a tumor suppressor gene, has been found damaged or deficient in many cancer (33-35). It may be one of the targets for anticancer treatment. Thus, we investigated whether PTEN is involved in the anti-proliferation effect of Res in human colon cancer cells. The PCR and western blot results show that Res can induce the expression of PTEN concentration-dependently (Fig. 4A and B). The PTEN inhibitor attenuates the anti-proliferation effect of Res in HCT116 cells (Fig. 4C). Exogenous expression of PTEN potentiates the proliferation inhibitory effect of Res, while knockdown of PTEN inhibits this effect of Res in HCT116 cells (Fig. 4D). These results indicate that PTEN is involved in the anti-proliferation of Res in human colon cancer cells.

Resveratrol downregulates PI3K/Akt signaling in HCT116 cells through upregulating PTEN. One major function of PTEN is to negatively regulate PI3K/Akt signaling. The $\mathrm{PI}$ KK/Akt signaling pathway is one of the essential pathways for cell survival and proliferation, which is over-activated in many human cancers, such as breast, colon and prostate cancers $(10,14)$. Thus, we investigated whether PI3K/Akt signaling is involved in the anti-proliferation effect of Res in HCT116 cells. The results indicated that Res has no apparent effect on the mRNA expression of Akt1 and Akt2 (Fig. 5A), but can reduce the phosphorylation level of Akt1/2 concentrationdependently (Fig. 5B). Res combined with adenovirus mediated exogenous expression of PTEN decreases the phosphorylation of Akt1/2 substantially (Fig. 5C), while knockdown of PTEN reverses the effect of Res on the phosphorylation of Akt1/2 (Fig. 5D). These results suggest that Res can inhibit PI3K/Akt signaling activation in HCT116 cells through the upregulation of PTEN.

Resveratrol inhibits Wnt/ $\beta$-catenin signaling transduction independent of PTEN/PI3K/Akt in HCT116 cells. The abnormal activation of $\mathrm{Wnt} / \beta$-catenin signaling is one of the major causes of cancer, which can be regulated by PTEN/ PI3K/Akt signaling through the phosphorylation of GSK-3 $\beta$. HCT116 cells are predisposed to the mutation of $\beta$-catenin, thus, it can not be degradated by the degradative complex in the canonical Wnt signaling pathway. We measured whether Res could still decrease the level of $\beta$-catenin in HCT116 cells. We found that Res has no apparent effect on the total level of GSK-3 $\beta$, but decreases the phosphorylation of GSK-3 $\beta$ substantially, as well as the protein level of $\beta$-catenin (Fig. 6A). The $\beta$-catenin/Tcf4 reporter assay results indicate 

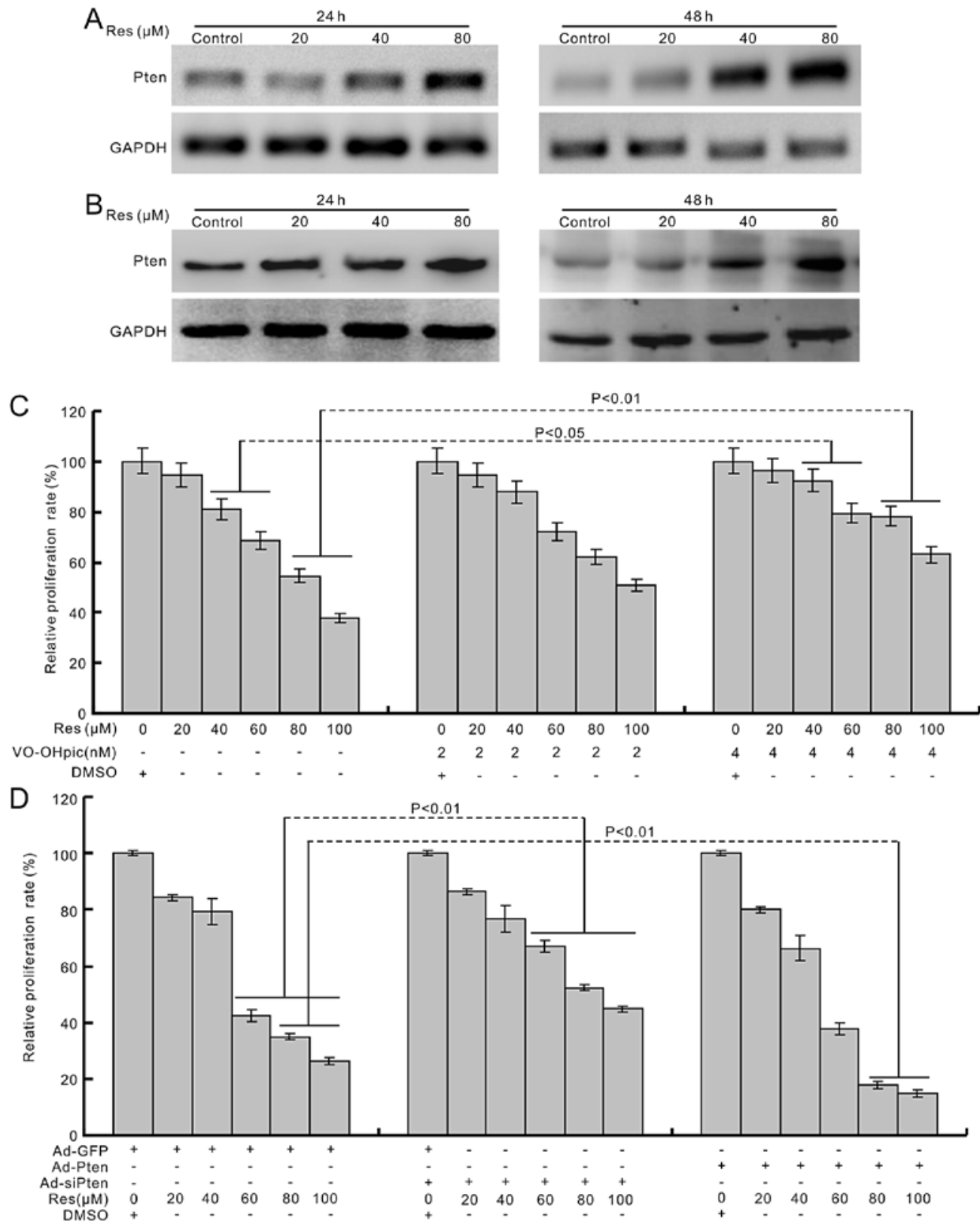

Figure 4. PTEN participates in the anti-proliferation effect of resveratrol (Res) in HCT116 cells. (A) Res upregulates the mRNA expression of PTEN. HCT116 cells were treated with different concentrations of Res or solvent. Total RNA were extracted at the indicated time point, followed by RT-PCR assay as described in Materials and methods. (B) Res increases the protein level of PTEN. HCT116 cells were treated with different concentrations of Res or solvent. Total protein was harvested at the indicated time point, followed by western blot assay as described in Materials and methods. GAPDH was used as loading control. (C) PTEN specific inhibitor partly reverses the anti-proliferation activities of Res in HCT116 cells. Cells were seeded in 24-well plates, treated with different concentrations of Res or combined with PTEN inhibitor (2 or $4 \mathrm{nM}$ ). (D) Exogenous expression or knockdown of PTEN affects the anti-proliferation activities of Res in HCT116 cells. Cells were seeded in 24-well plates, treated with different concentrations of Res or combined with infection of corresponding recombinant adenovirus (Ad-PTEN or Ad-siPTEN). The crystal violet stain and quantification were performed as described in Materials and methods. Each condition was done in triplicate.

that Res inhibited the reporter activity concentrationdependently (Fig. 6B). Moreover, the $\beta$-catenin mRNA expression was also decreased by Res (Fig. 6C). These results suggest that the canonical Wnt signaling pathway is involved in the anti-proliferation effect of Res in HCT116 cells.

\section{Discussion}

Colon cancer is one of the most frequently diagnosed malignancies, with high incidence in western countries (1). Although the treatment for colon cancer has advanced substantially, the prognosis is still more modest than had been hoped (36). There is a great clinical need to explore new treatment regimens for colon cancer. In this investigation, we demonstrated that Res has potent anti-proliferation activity in human colon cancer cells, and the anti-proliferation effect of Res may be mediated by inhibiting PI3K/Akt signaling through upregulating the expression of PTEN and blocking Wnt/ $\beta$-catenin signaling transduction, respectively.

Res, as a natural polyphenolic compound, is found in the skin of red grapes and other fruits as well as in Japanese 

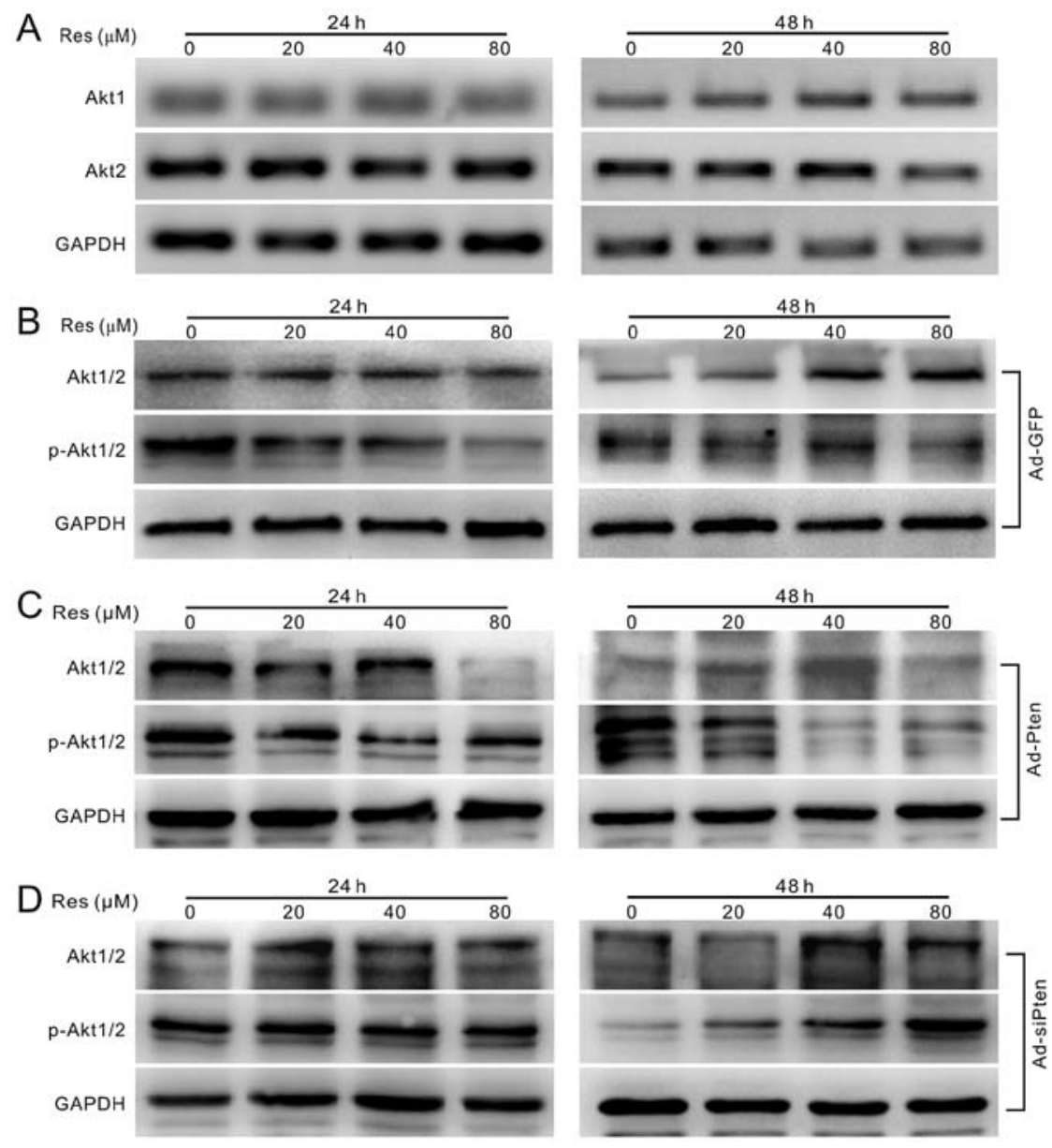

Figure 5. Resveratrol (Res) inhibits PI3K/Akt signaling in HCT116 cells. (A) The effect of Res on the mRNA expression of Akt1 and Akt2. HCT116 cells were treated with different concentrations of Res or solvent, total RNA was harvested and RT-PCR performed to detect the expression of mRNA as described in Materials and methods. (B) Res decreases the phosphorylation of Akt1/2. HCT116 cells were treated with different concentrations of Res or solvent, combined with Ad-GFP. Total protein was harvested at the indicated time point, followed by western blot assay as described in Materials and methods. (C) Exogenous expression of PTEN enhances the inhibition of PI3K/Akt signaling induced by Res. HCT116 cells were treated with different concentrations of Res or solvent, combined with exogenous expression of PTEN (mediated by Ad-PTEN). Total protein was harvested at the indicated time point, followed by western blot assay as described in Materials and methods. (D) Knockdown of PTEN reverses the inhibition of PI3K/Akt signaling induced by Res. HCT116 cells were treated with different concentrations of Res or solvent, combined with recombinant adenovirus Ad-siPTEN. Total protein was harvested at the indicated time point, followed by western blot assay as described in Materials and methods. GAPDH was used as loading control. Each condition was done in triplicate.

knotweed roots (11). Several studies have proved that Res can inhibit proliferation and induce apoptosis of breast cancer cells, prostate cancer cells and colon cancer cells $(10,13,14)$. No clinical trial has yet reported the cancer prevention effect of Res (37). Moreover, the bioavailability of Res is very low, so even a high dose of Res may not reach the sufficient concentration required for systemic treatment for cancers (38), however, this may be a benefit for digestive tract cancer treatment. Our in vitro results show that Res can inhibit the proliferation of HCT116 cells, even at the concentration of $20 \mu \mathrm{M}$. These data confirmed that Res has the potential to be an anticancer agent. Interestingly, our investigation indicates that Res shows no apparent proliferation inhibitory effect in HEK-293 cells, even at the concentration of $100 \mu \mathrm{M}$ (data not shown). This result implies that the anti-proliferation effect of Res may be more specific to cancer cells.

The above studies, and our new results validate the proliferation inhibitory effect of Res on colon cancer cells, but the exact molecular mechanism underlying this remains unknown. Vanamala et al reported that Res could induce apoptosis through the suppression of Wnt pathway and activa- tion of p53 signaling pathways in human colon cancer (39). The expression of MicroRNA-21 participates in the inhibition of prostate cancer growth and metastasis initialized by Res (16). Recent studies indicate that $\mathrm{p} 38$ and PI3K signaling pathways are involved in the anticancer activity of Res (40). Wnt, PI3K/Akt and p38 signaling pathways are all essential for cell proliferation and differentiation, and found to be aberrant in many cancers. However, the exact molecular mechanism of how Res regulates these signaling pathways remains unclear.

PTEN, a tumor suppressor often mutated or lost in many cancers, acts as a phosphatase to specifically catalyse PIP3 dephosphorylation at the 3-phosphate of the inositol ring and turn PIP3 to PIP2 through which it negatively regulates PI3K/ Akt signaling. PI3K/Akt signaling has been associated with many cellular functions, including proliferation, differentiation, motility and survival. Previous studies indicated that PTEN may play an important role in early stages of sporadic colorectal carcinogenesis and reduced or lost PTEN expression is more frequent in colon cancer (25); colon cancer cells with high expression of PTEN is correlated with chemo- 

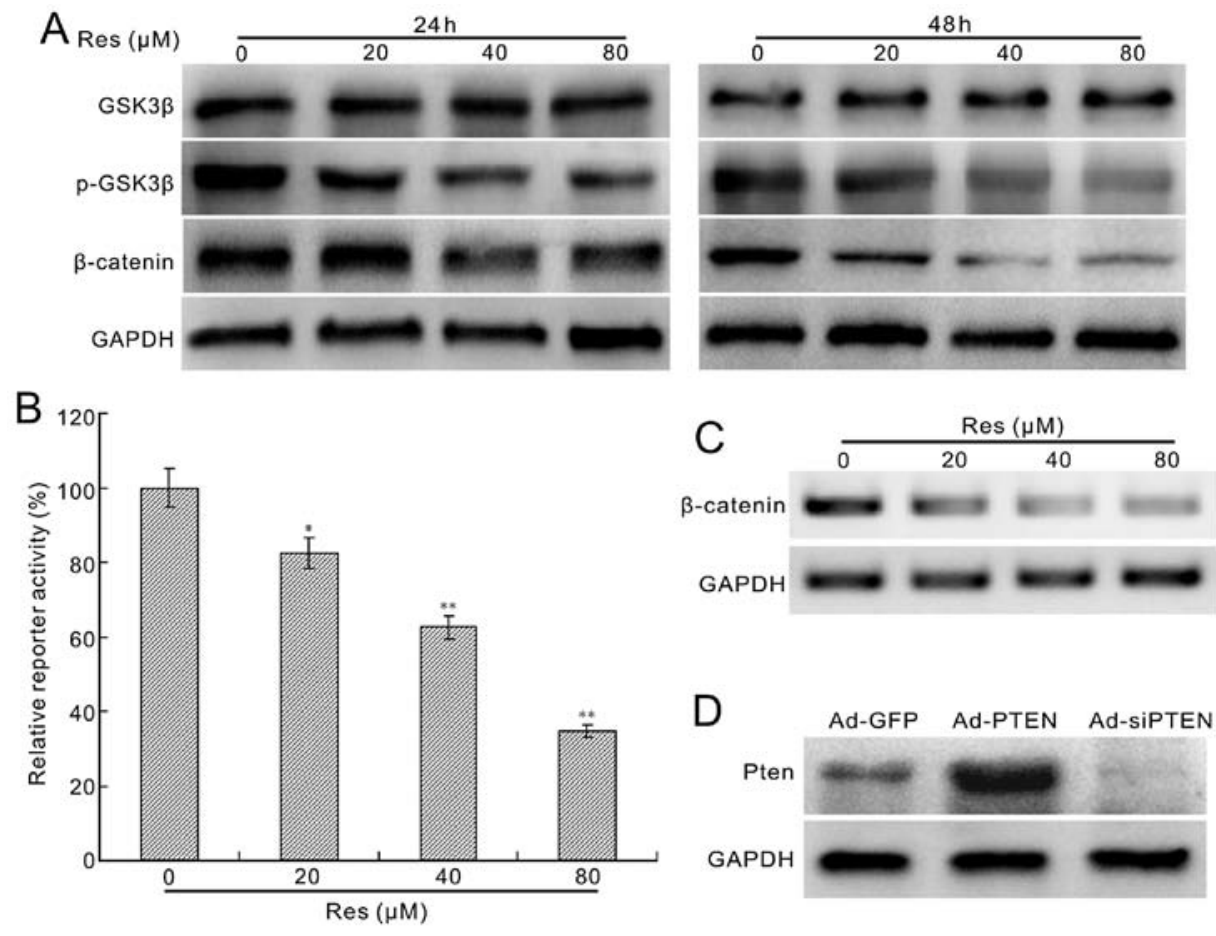

Figure 6. Resveratrol (Res) inhibits Wnt/ $\beta$-catenin signaling transduction in HCT116 cells. (A) Res decreases the phosphorylation of GSK-3 $\beta$ and the protein level of $\beta$-catenin. HCT116 cells were treated with different concentrations of Res or solvent. Total protein was harvested at the indicated time point, followed by western blot assay as described in Materials and methods. (B) Res inhibits the $\beta$-catenin/Tcf reporter activity. Cells were seeded in T25 flasks and transfected with $3.0 \mu \mathrm{g}$ per flask of pTOP-Luc ( $\beta$-catenin/TCF4 reporter plasmid) using Lipofectamine. After $12 \mathrm{~h}$, cells were replated in $24-w e l l$ plates and treated with different concentrations of Res or solvent as control. After $24 \mathrm{~h}$, cells were lysed and subjected to luciferase activity assays. ${ }^{*} \mathrm{P}<0.05$ vs. control group; ${ }^{* *} \mathrm{P}<0.01$ vs. control group. (C) Res decreases the mRNA expression of $\beta$-catenin. HCT116 cells were treated with different concentrations of Res or solvent for $24 \mathrm{~h}$. Total RNA was run on RT-PCR to detect the mRNA expression of $\beta$-catenin. (D) The effect of recombinant adenovirus on the expression of PTEN. HCT116 cells were seeded in a 6-well plate and infected with Ad-GFP, Ad-PTEN and Ad-siPTEN. After $24 \mathrm{~h}$, total protein was harvested for western blotting to measure the expression of PTEN. Each condition was done in triplicate.

sensitivity (41). Our results show that Res can upregulate the expression of PTEN in HCT116 cells. Either PTEN specific inhibitor or knockdown of PTEN can attenuate the anti-proliferation effect of Res in HCT116 cells, while exogenous expression of PTEN can potentiate the proliferation inhibitory effect of Res in these cells. These data indicate that PTEN may be important for the anti-proliferation effect of Res in HCT116 cells. Further analysis confirmed that the upregulated PTEN by Res is correlated with the inactivation of PI3K/Akt signaling by decreasing the phosphorylation of Akt1/2 in HCT116 cells. Therefore, our results suggest that PTEN/PI3K/Akt is involved in the proliferation inhibitory effect of Res in HCT116 cells.

Another major cause of colon cancer is the over activated Wnt/ $\beta$-catenin signaling pathway. Most colon cancer cells are predisposed to the mutation of $\beta$-catenin or APC (42). Wnt signaling, including canonical and noncanonical Wnt signaling, plays an important role in embryogenesis and development. The $\beta$-catenin plays a pivotal role in canonical Wnt signaling. In the absence of Wnt proteins, Axin, GSK-3 $\beta$ and APC assemble as a complex to promote the proteolytic degradation of $\beta$-catenin. When Wnt proteins bind with the frizzled receptor, the degradation complex will be destroyed and the $\beta$-catenin can be accumulated in the cytoplasm and translocate to the nucleus. Eventually, $\beta$-catenin interacts with TCF/LEF transcription factors to regulate the downstream gene expression.
The mutation of APC, $\beta$-catenin or phosphorylation of GSK-3 $\beta$ cause $\beta$-catenin not to be degraded normally by the destruction complex and accumulate in the cytoplasm. The activation of PI3K/Akt can activate the canonical Wnt signaling through the phosphorylation of GSK-3 $\beta$ by the phosphorylated Akt1/2, blocking the formation of $\beta$-catenin destroying complex (28). Hence, the upregulation of PTEN may inhibit the canonical Wnt signaling by promoting the degradation of $\beta$-catenin. Although our results show that Res can decrease the phosphorylation of GSK-3 $\beta$, the protein level of $\beta$-catenin, and the $\beta$-catenin/Tcf4 reporter activity, but it may not result from the decreased phosphorylation of GSK-3 $\beta$ by PTEN/PI3K/Akt signaling. As it is predisposed to mutation of $\beta$-catenin in HCT116 cells, the $\beta$-catenin can not be degraded by the destruction complex in this colon cancer cells. With additional investigation, we unveiled that Res can inhibit the mRNA expression of $\beta$-catenin. Thus, these results suggest that Res can inhibit the Wnt/ $\beta$-catenin signaling transduction, but it may not result from the upregulation of PTEN in HCT116 cells.

Taken together, Our data strongly suggest that Res can inhibit the proliferation and promote apoptosis in colon cancer cells. These activities of Res may be mediated by PI3K/Akt signaling through upregulating the expression of PTEN and reducing the $\mathrm{Wnt} / \beta$-catenin signaling transduction through inhibiting the expression of $\beta$-catenin, respectively. However, the detailed molecular mechanism of how Res regu- 
late the expression of PTEN and $\beta$-catenin need to be further deciphered.

\section{Acknowledgements}

We thank Dr Bert Vogelstein of the Johns Hopkins Oncology Center (Baltimore, MD, USA) for his kind provision of HCT116 cells. We thank Professor Qi-Xin Zhou of Chongqing Medical University (Chongqing, China) for his critical reading of the manuscript. This study was supported in part by research grants from Natural Science Foundation of China (Grants: NSFC 81071462 and 81372120), Chongqing Science \& Technology Commission of China (Grant: CSTC 2011BB5129), and the National Basic Research Program of China (Grant: 2011CB70790).

\section{References}

1. Brunagel G, Vietmeier BN, Bauer AJ, Schoen RE and Getzenberg RH: Identification of nuclear matrix protein alterations associated with human colon cancer. Cancer Res 62: 2437-2442, 2002.

2. Fey MF: Adjuvant therapy for colon cancer. Schweiz Med Wochenschr 130: 1760-1765, 2000 (In German).

3. Cragg GM, Grothaus PG and Newman DJ: Impact of natural products on developing new anti-cancer agents. Chem Rev 109 3012-3043, 2009.

4. Mishra BB and Tiwari VK: Natural products: an evolving role in future drug discovery. Eur J Med Chem 46: 4769-4807, 2011.

5. Zou DM, Brewer M, Garcia F, et al: Cactus pear: a natural product in cancer chemoprevention. Nutr J 4: 25, 2005.

6. Konkimalla VB and Efferth T: Anti-cancer natural product library from traditional Chinese medicine. Comb Chem High Throughput Screen 11: 7-15, 2008

7. Khan N, Afaq F and Mukhtar H: Cancer chemoprevention through dietary antioxidants: progress and promise. Antioxid Redox Signal 10: 475-510, 2008.

8. Korkina LG, De Luca C, Kostyuk VA and Pastore S: Plant polyphenols and tumors: from mechanisms to therapies, prevention, and protection against toxicity of anti-cancer treatments. Curr Med Chem 16: 3943-3965, 2009.

9. Das S, Santani DD and Dhalla NS: Experimental evidence for the cardioprotective effects of red wine. Exp Clin Cardiol 12: 5-10, 2007.

10. Jang M, Cai L, Udeani GO, et al: Cancer chemopreventive activity of resveratrol, a natural product derived from grapes. Science 275: 218-220, 1997.

11. Gehm BD, McAndrews JM, Chien PY and Jameson JL: Resveratrol, a polyphenolic compound found in grapes and wine, is an agonist for the estrogen receptor. Proc Natl Acad Sci USA 94: 14138-14143, 1997.

12. Bhat KPL, Kosmeder JW II and Pezzuto JM: Biological effects of resveratrol. Antioxid Redox Signal 3: 1041-1064, 2001.

13. Gulvady AA, Ciolino HP, Cabrera RM and Jolly CA: Resveratrol inhibits the deleterious effects of diet-induced obesity on thymic function. J Nutr Biochem 24: 1625-1633, 2013.

14. Fang JY, Li ZH, Li Q, Huang WS, Kang L and Wang JP Resveratrol affects protein kinase $\mathrm{C}$ activity and promotes apoptosis in human colon carcinoma cells. Asian Pac J Cancer Prev 13: 6017-6022, 2012.

15. Lu R and Serrero G: Resveratrol, a natural product derived from grape, exhibits antiestrogenic activity and inhibits the growth of human breast cancer cells. J Cell Physiol 179: 297-304, 1999.

16. Sheth S, Jajoo S, Kaur T, et al: Resveratrol reduces prostate cancer growth and metastasis by inhibiting the Akt/MicroRNA-21 pathway. PLoS One 7: e51655, 2012.

17. Fouad M, Agha A, Merzabani MA and Shouman S: Resveratrol inhibits proliferation, angiogenesis and induces apoptosis in colon cancer cells: Calorie restriction is the force to the cytotoxicity. Hum Exp Toxicol 32: 1067-1080, 2013.

18. Yang HL, Chen WQ, Cao X, et al: Caveolin-1 enhances resveratrolmediated cytotoxicity and transport in a hepatocellular carcinoma model. J Transl Med 7: 22, 2009.
19. Miura D, Miura Y and Yagasaki K: Hypolipidemic action of dietary resveratrol, a phytoalexin in grapes and red wine, in hepatoma-bearing rats. Life Sci 73: 1393-1400, 2003.

20. Liu HS, Pan CE, Yang W and Liu XM: Antitumor and immunomodulatory activity of resveratrol on experimentally implanted tumor of $\mathrm{H} 22$ in Balb/c mice. World J Gastroenterol 9: 1474-1476, 2003.

21. Fresno Vara JA, Casado E, de Castro J, Cejas P, Belda-Iniesta C and Gonzalez-Baron M: PI3K/Akt signalling pathway and cancer. Cancer Treat Rev 30: 193-204, 2004.

22. Mahimainathan L and Choudhury GG: Inactivation of plateletderived growth factor receptor by the tumor suppressor PTEN provides a novel mechanism of action of the phosphatase. J Biol Chem 279: 15258-15268, 2004.

23. Takeda K, Kanekura T and Kanzaki T: Negative feedback regulation of phosphatidylinositol 3-kinase/Akt pathway by overexpressed cyclooxygenase-2 in human epidermal cancer cells. J Dermatol 31: 516-523, 2004.

24. Chalhoub N and Baker SJ: PTEN and the PI3-kinase pathway in cancer. Annu Rev Pathol 4: 127-150, 2009.

25. Waniczek D, Snietura M, Mlynarczyk-Liszka J, et al: PTEN expression profiles in colorectal adenocarcinoma and its precancerous lesions. Pol J Pathol 64: 15-20, 2013.

26. Franke TF, Kaplan DR, Cantley LC and Toker A: Direct regulation of the Akt proto-oncogene product by phosphatidylinositol-3,4-bisphosphate. Science 275: 665-668, 1997.

27. Zhang W, Zhang H, Wang N, et al: Modulation of $\beta$-catenin signaling by the inhibitors of MAP kinase, tyrosine kinase, and PI3-kinase pathways. Int J Med Sci 10: 1888-1898, 2013.

28. Robertson BW and Chellaiah MA: Osteopontin induces betacatenin signaling through activation of Akt in prostate cancer cells. Exp Cell Res 316: 1-11, 2010.

29. He BC, Chen L, Zuo GW, et al: Synergistic antitumor effect of the activated PPARgamma and retinoid receptors on human osteosarcoma. Clin Cancer Res 16: 2235-2245, 2010.

30. Stepanovic S, Vukovic D, Dakic I, Savic B and SvabicVlahovic M: A modified microtiter-plate test for quantification of staphylococcal biofilm formation. J Microbiol Methods 40: $175-179,2000$.

31. He TC, Zhou S, da Costa LT, Yu J, Kinzler KW and Vogelstein B: A simplified system for generating recombinant adenoviruses. Proc Natl Acad Sci USA 95: 2509-2514, 1998.

32. Joe AK, Liu H, Suzui M, Vural ME, Xiao D and Weinstein IB Resveratrol induces growth inhibition, S-phase arrest, apoptosis, and changes in biomarker expression in several human cancer cell lines. Clin Cancer Res 8: 893-903, 2002.

33. Pal I and Mandal M: PI3K and Akt as molecular targets for cancer therapy: current clinical outcomes. Acta Pharmacol Sin 33: 1441-1458, 2012.

34. Lin MS, Huang JX, Chen WC, et al: Expression of PPARgamma and PTEN in human colorectal cancer: An immunohistochemical study using tissue microarray methodology. Oncol Lett 2: 1219-1224, 2011.

35. Garcia JM, Silva JM, Dominguez G, et al: Allelic loss of the PTEN region (10q23) in breast carcinomas of poor pathophenotype. Breast Cancer Res Treat 57: 237-243, 1999.

36. Aggarwal $\mathrm{S}$ and Chu E: Current therapies for advanced colorectal cancer. Oncology 19: 589-595, 2005.

37. Athar M, Back JH, Tang X, et al: Resveratrol: a review of preclinical studies for human cancer prevention. Toxicol Appl Pharmacol 224: 274-283, 2007.

38. Boocock DJ, Faust GE, Patel KR, et al: Phase I dose escalation pharmacokinetic study in healthy volunteers of resveratrol, a potential cancer chemopreventive agent. Cancer Epidemiol Biomarkers Prev 16: 1246-1252, 2007.

39. Vanamala J, Reddivari L, Radhakrishnan S and Tarver C: Resveratrol suppresses IGF-1 induced human colon cancer cell proliferation and elevates apoptosis via suppression of IGF-1R/ Wnt and activation of p53 signaling pathways. BMC Cancer 10: $238,2010$.

40. Gweon EJ and Kim SJ: Resveratrol induces MMP-9 and cell migration via the p38 kinase and PI-3K pathways in HT1080 human fibrosarcoma cells. Oncol Rep 29: 826-834, 2013.

41. Hsu CP, Kao TY, Chang WL, Nieh S, Wang HL and Chung YC: Clinical significance of tumor suppressor PTEN in colorectal carcinoma. Eur J Surg Oncol 37: 140-147, 2011.

42. He BC, Gao JL, Luo X, et al: Ginsenoside Rg3 inhibits colorectal tumor growth through the down-regulation of $\mathrm{Wnt} / \beta$-catenin signaling. Int J Oncol 38: 437-445, 2011. 